\title{
The pharmacological challenges of the perinatal period
}

\author{
Srilakshmi Pingali \\ Associate Professor, Gandhi Medical College, Secunderabad, Telangana, India
}

*Corresponding Author: Srilakshmi Pingali

Email: drpingali@gmail.com

The perinatal period starting from conception to the first year postpartum is a challenging time for the psychiatrist. The psychiatrist is responsible for the wellbeing of both mother and the baby. With the stigma surrounding the use of psychotropic medication not only in the general public and but also in our medical fraternity, the challenge increases manifold. ${ }^{1}$

The various challenges faced by the treating psychiatrist in the perinatal period are either a new onset psychiatric illness during pregnancy, managing a preexisting psychiatric illness in pregnancy, risk of medication on the developing fetus and the balancing act of whether or not to continue with the medication. With increasing knowledge of long term effects of untreated psychiatric illness on the baby the decision becomes more challenging.

A good working knowledge of the physiological changes in pregnancy and their consequent effects on the pharmacokinetics of the drugs administered is therefore helpful in the dose of the drugs to be administered. For instance the recurrent nausea in pregnancy accompanied by reduced gastric emptying leads to reduced oral absorption of the drugs. The increased body water leading to increased volume of distribution of the drug leads to lowered serum plasma levels and at the same time reduced plasma albumin leads to reduced protein binding of the drugs which increases its free active level. Similarly an increased cardiac output during pregnancy leads to an increased renal blood flow, which in turn increases the renal clearance of the drugs. This is especially of significance in drugs like lithium whose major route of clearance is the renal clearance. The change in activity of the hepatic enzymes also lead to altered drug metabolism which in turn increase or decrease the blood levels of the drug. ${ }^{2}$ So do we then increase the dose of the drug? The answer surely is a no as we do not treat the blood levels but the patient. Hence treating the emerging symptoms and adjusting doses keeping the altered pharmacokinetics in mind is helpful.

Another factor while changing the drug dose in the mother is the fetus's ability to metabolize the drug. Low levels of drug binding to plasma proteins, immature hepatic metabolism, relatively permeable blood brain barrier and difficulties in transporting drug metabolites back to the maternal circulation produce higher drug concentrations in fetal than maternal blood. ${ }^{3}$ So any dose change in the mother leads to increased doses in the fetal circulation, a point to be kept in mind while making dose changes.

Various psychiatric disorders tend to relapse more during pregnancy. For instance bipolar disorder has a $50 \%$ chance of relapse during pregnancy. The risk is 2.3 times higher after discontinuation of the mood stabilizer. The risk of relapse is highest in the first 4 weeks postpartum. ${ }^{4}$ Depression in pregnancy is a strong predictor of postpartum depression and women who were previously depressed and discontinued medication are 5 times more likely to relapse. In fact $25 \%$ relapse even while on medication. Untreated psychiatric illness leads to complications like low birth weight, preterm delivery, infant neglect and lack of mother child bonding. 5

Untreated psychiatric illness is also known to effect the long term growth of the child. Studies have shown that if mother is stressed while pregnant, her child is substantially more likely to have emotional or cognitive problems, including an increased risk of attentional deficit/hyperactivity, anxiety and language delay. ${ }^{6}$

All this seem to weigh the balance in favor of using medication during the perinatal period. However certain do's and don'ts should be kept in mind. Polypharmacy, rapid discontinuation and unnecessary switching of a drug in search of a safer alternative there by exposing the fetus to more than one drug should be avoided. It should be kept in mind that the adverse effects of the drug depend on the time of exposure, dose and the teratogenic potential of the drugs. A drug is said to have a teratogenic potential only if it has risk of causing physical deformities over the base line rate of 2.0 $2.5 \% .{ }^{4}$ Most drugs used in psychiatry except for drugs like sodium valproate and carbamazepine fall below this value. ${ }^{7}$

Therefore before starting a drug during the perinatal period it is necessary to evaluate the severity of the disorder. Mild depression emerging for the first time in pregnancy can very well be managed by non-pharmacological methods. ${ }^{8}$ Attitudes and wishes of the patient are paramount while taking decision regarding medication in pregnancy and the need to document any such discussion cannot be overemphasized.

Perinatal psychiatry however does not confine itself to pharmacological treatment alone but is concerned with dealing with the mother baby dyad as a whole. Starting from pre pregnancy counselling to reducing associated risk factors like obesity, substance abuse and comorbid medical illnesses which add to fetal morbidity form a part of the treatment plan. ${ }^{9}$ An ideal scenario is a planned pregnancy in our patients. In such a scenario, the associated risk factors can be addressed and the drugs continued after discussion and documentation. However such ideal case scenarios rarely exist. Hence treatment of each patient has to be individualized. 
A working knowledge of the teratogenic potential of the drugs and the pharmacokinetic changes they undergo in pregnancy, liaising with the treating team like the obstetrician and the pediatrician and documentation of the collective decision taken with the patient and family are surely the tenets on which perinatal psychiatry functions.

\section{References}

1. "Psychiatry is not a science like others" - a focus group study on psychotropic prescribing in primary care. Hedenrud TM, Svensson SA, Wallerstedt SM. BMC Fam Pract. 2013 Aug $12 ; 14$.

2. Physiologic and pharmacokinetic changes in pregnancy. Maged M. Costantine. Front. Pharmacol. 03 April 2014

3. Babu GN, Desai G, Chandra PS.Antipsychotics in pregnancy and lactation. Indian J Psychiatry. 2015;57:303-307.

4. Mood stabilizers in pregnancy and lactation. Sandeep Grover and Ajit Avasthi. Indian J Psychiatry. 2015;57(Suppl 2):S308S323.
5. Perinatal depression: pathogenesis and treatment - MeltzerBrody. Dialogues Clin Neurosci. 2011;13(1).

6. Satyanarayana VA, Lukose A, Srinivasan K. Maternal mental health in pregnancy and child behavior. Indian J Psychiatry. 2011;53:351-361.

7. Pharmacotherapy for Mood Disorders in Pregnancy: A Review of Pharmacokinetic Changes and Clinical Recommendations for Therapeutic Drug Monitoring.

8. Guidelines for the management of depression during pregnancy. Deborah. R. Kim, ohn P. O' Reardon, C. Neill Epperson. Curr Psychiatry Rep. 2010;12(4):279-281.

9. Dere SS. IPS travel fellowship in perinatal psychiatry. Indian $J$ Psychiatry. 2017;59:402-403. 